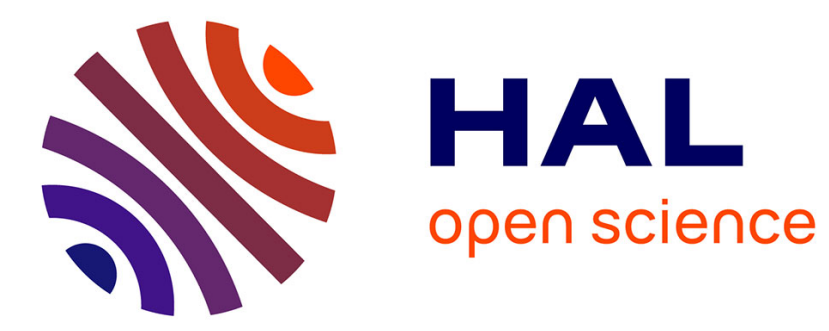

\title{
Unipolar and Bipolar High-Magnetic-Field Sensors Based on Surface Acoustic Wave Resonators
}

V. Polewczyk, K. Dumesnil, D. Lacour, M. Moutaouekkil, H. Mjahed, Nicolas Tiercelin, S. Petit-Watelot, H. Mishra, Yannick Dusch, S. Hage-Ali, et al.

\section{- To cite this version:}

V. Polewczyk, K. Dumesnil, D. Lacour, M. Moutaouekkil, H. Mjahed, et al.. Unipolar and Bipolar High-Magnetic-Field Sensors Based on Surface Acoustic Wave Resonators. Physical Review Applied, 2017, 8 (2), 10.1103/PhysRevApplied.8.024001 . hal-01686932

\section{HAL Id: hal-01686932 \\ https://hal.univ-lorraine.fr/hal-01686932}

Submitted on 17 Jan 2018

HAL is a multi-disciplinary open access archive for the deposit and dissemination of scientific research documents, whether they are published or not. The documents may come from teaching and research institutions in France or abroad, or from public or private research centers.
L'archive ouverte pluridisciplinaire HAL, est destinée au dépôt et à la diffusion de documents scientifiques de niveau recherche, publiés ou non, émanant des établissements d'enseignement et de recherche français ou étrangers, des laboratoires publics ou privés. 


\title{
Unipolar and Bipolar High-Magnetic-Field Sensors Based on Surface Acoustic Wave Resonators
}

\author{
V. Polewczyk, ${ }^{1}$ K. Dumesnil, ${ }^{1}$ D. Lacour, ${ }^{1}$ M. Moutaouekkil, ${ }^{1}$ H. Mjahed, ${ }^{1}$ N. Tiercelin, ${ }^{2}$ S. Petit Watelot, ${ }^{1}$ \\ H. Mishra, ${ }^{1}$ Y. Dusch, ${ }^{2}$ S. Hage-Ali, ${ }^{1}$ O. Elmazria, ${ }^{1}$ F. Montaigne, ${ }^{2}$ A. Talbi, ${ }^{2}$ \\ O. Bou Matar, ${ }^{2}$ and M. Hehn ${ }^{1}$ \\ ${ }^{1}$ Institut Jean Lamour, Université de Lorraine CNRS, \\ UMR 7198, BP 70239, F-54506 Vandoeuvre lès Nancy, France \\ ${ }^{2}$ IEMN, UMR CNRS 8520, PRES Lille Nord de France, EC Lille, 59651 Villeneuve d'Ascq, France
}

(Received 20 April 2017; published 1 August 2017)

\begin{abstract}
While surface acoustic wave (SAW) sensors have been used to measure temperature, pressure, strains, and low magnetic fields, the capability to measure bipolar fields and high fields is lacking. In this paper, we report magnetic surface acoustic wave sensors that consist of interdigital transducers made of a single magnetostrictive material, either $\mathrm{Ni}$ or $\mathrm{TbFe}_{2}$, or based on exchange-biased $(\mathrm{Co} / \mathrm{IrMn})$ multilayers. By controlling the ferromagnet magnetic properties, high-field sensors can be obtained with unipolar or bipolar responses. The issue of hysteretic response of the ferromagnetic material is especially addressed, and the control of the magnetic properties ensures the reversible behavior in the SAW response.
\end{abstract}

DOI: 10.1103/PhysRevApplied.8.024001

\section{INTRODUCTION}

Surface acoustic wave (SAW) devices are of major interest in sensor applications due to their ease of manufacturing, sensitivity, small size, and wireless interrogation [1-4]. Indeed, especially in the SAW resonator geometry [1-4], the sensor can be wireless addressed and measured without any embedded power source. SAW sensors have been used up to now to measure a large variety of stimuli like temperature, pressure, strain, and the presence of chemicals $[5,6]$. It is also known that the acoustic wave velocity or the resonant frequency of SAW resonators including a magnetostrictive material can be changed by applying a magnetic field. Indeed, the elastic properties of magnetoelastic materials can greatly depend on the magnetic state of the sample and, thus, on the applied magnetic field. By using single magnetostrictive materials or composites with enhanced magnetoelectric coefficients, magnetic SAW (MSAW) sensors have been proposed during the last ten years [7-10]. Proofs of concept have been proposed by combining either $\mathrm{Ni}$ [9-11], amorphous $\mathrm{TbFe}_{2}$ [12], or $(\mathrm{CoFe} / \mathrm{CoTb})$ multilayers [13] with $\mathrm{LiNbO}_{3}$ (LNO) or quartz substrates. As far as the geometry is concerned, most of the devices are based on magnetic thin films deposited in the gap between the input and output interdigital transducers (IDTs) of a delay line [12], while the use of the SAW resonator remains confidential $[9,10]$.

Despite these various works, one can underline that some important aspects have been largely ignored concerning both the fundamental understanding of the SAW magnetoacoustic response and the requirements to develop efficient field sensors. Many studies report the SAW response without clear indication regarding the film or the IDT's magnetic properties, while this is crucial to understand and control the SAW response. The exact roles of the magnetic anisotropy, the dynamic electromechanical coupling, or the propagation direction are, for example, still debated, as shown by the variety of interpretations proposed for MSAW response [9,11-13]. The majority of works also focused on the acoustic response for magnetic fields applied in the plane of the device, neglecting the case where the field is applied perpendicular to the plane and its link to the magnetic behavior in this configuration. Another important lack concerns the incidence of magnetic hysteresis on the SAW magnetoacoustic response and the appearance of hysteresis in the SAW response itself, while the latter can limit the use as a field sensor. Finally, the use of SAW devices to sense magnetic fields has been restricted up to now to low magnetic fields, while the capability to wirelessly measure bipolar fields and/or high fields is still lacking.

In this paper, we report magnetic SAW devices that consist of IDTs made of either textured $\mathrm{Ni}$ or $\mathrm{Co} / \mathrm{IrMn}$, and crystalline $\mathrm{TbFe}_{2}$ magnetostrictive materials. $\mathrm{Ni}$ is chosen because of its previous use for demonstration (with magnetostriction coefficients of $\lambda_{111}=-27.10^{-6}$ and $\lambda_{100}=-64.10^{-6}$ at room temperature) [14]. We report the attempt of SAW structures using crystalline $\mathrm{TbFe}_{2}$ that exhibit record saturation magnetostriction in bulk form $\left(\lambda_{111}=2460.10^{-6}\right.$ and $\lambda_{100}=100.10^{-6}$ at room temperature) [15] and high coercive fields [16] for high-field sensing. For sensing magnetic fields perpendicular to the device, we show that the response of the in-plane magnetization component has to be tailored to remove magnetization flips while keeping its rotation. We show the use of complex architectures based on exchange-biased (Co/IrMn) multilayers tested in SAW structures. The association of a ferromagnetic layer (Co) and an antiferromagnetic layer 
(IrMn), indeed, induces an exchange-bias field equivalent to a first-order anisotropy axis that permits us to control the rotation of the in-plane magnetization component. IrMn is chosen for its ease to obtain the (111) texture, its high interfacial exchange coupling, and moderate blocking temperature $\left(T_{B}=200^{\circ} \mathrm{C}\right)$ [17]. While the magnetostriction at the saturation of polycrystalline Co is around $-50.10^{-6}$ at room temperature [18], no data can be found for IrMn.

The combined use of all these materials allows us to show that the three-dimensional configuration of the magnetization in the IDT's fingers or in the ferromagnetic layer is of prime importance to understand and reproduce the MSAW response, while this characterization is lacking in many publications on MSAW. We demonstrate that not only the projection of the magnetization along the field direction is important to understand the MSAW response but also the component perpendicular to the applied field. Therefore, a device that does not appear hysteretic in conventional magnetometry can present hysteresis in the SAW response. Our understanding and control of the magnetic response leads us to propose three advanced characteristics for MSAW sensors. First of all, we show that with the proper choice of magnetic material, a bipolar field sensor can be built with a MSAW sensor, i.e., a sensor that can measure not only the intensity of the field but also the fact that the field is positive or negative.

Second, we show that MSAW is able to measure magnetic fields for geometries with the field applied perpendicular to substrate plane without any hysteresis. This geometry is rarely reported, and the response is not analyzed on the basis of the magnetic response of the magnetic wires. This measurement configuration extends the use of MSAW devices restricted up to now to the in-plane applied-field geometries. Finally, in the SAW resonator geometry, we show that the shape of the MSAW fingers plays a significant role in the magnetic anisotropy, especially for soft magnetic materials. Engineering the IDT's shape permits us to tailor and control the MSAW field response. Up to now, the MSAW responses could not be engineered since their responses depend on the properties of the films that have been used, which are difficult to change or adjust. Here, we show that keeping the material constant, the MSAW response can be adjusted just by changing the aspect ratio of the IDTs. This feature is fundamental to adjust the MSAW characteristics for dedicated applications.

\section{GROWTH OF THE MAGNETOSTRICTIVE MATERIAL}

As a first step, the growth of the magnetostrictive material is studied and optimized. This optimization especially concerns the $\mathrm{TbFe}_{2}$ thin layer that is grown on lithium niobate (LNO) substrates with the aim of benefitting from the reported record saturation magnetostriction [15]. In the literature, several orientations of LNO have been used to demonstrate acoustic wave propagation. However, the
LNO 41Y cut has been shown to give the highest velocity variation due to its huge electromechanical coupling factor [19]. We choose, therefore, to report in this paper only the results obtained for this substrate orientation, while additional information about growth or SAW response with different substrate orientations can be found elsewhere [20].

Ni-based and (Co/IrMn)-based devices are obtained by UHV magnetron sputtering. $\mathrm{LNO} / \mathrm{Ta}(5 \mathrm{~nm}) / \mathrm{Ni}(50 \mathrm{~nm}) /$ $\mathrm{Ta}(5 \mathrm{~nm})$ and $\mathrm{LNO} / \mathrm{Ta}(5 \mathrm{~nm}) /\left[\mathrm{Co}(5 \mathrm{~nm}) / \mathrm{Ir}_{20} \mathrm{Mn}_{80}\right.$ $(5 \mathrm{~nm})]_{15} / \mathrm{Pt}(5 \mathrm{~nm})$ heterostructures are grown at room temperature with operating $\mathrm{Ar}$ pressures fixed to $7.10^{-3}$ and $5.10^{-3}$ mbar for Ni-based and (Co/IrMn)-based devices, respectively. Before deposition, the LNO substrate is sputtered in order to promote the multilayer adhesion. The amorphous Ta layer in contact with LNO is usually used to favor adhesion of the top layers and also a (111) crystallographic texture. The Ta or Pt top layers protect the multilayers against oxidation and technological processes. In ( $\mathrm{Co} / \mathrm{IrMn})$ multilayers, the exchange field depends both on the Co and IrMn thicknesses. Its maximum is reached for IrMn thickness of $5 \mathrm{~nm}$ and varies inversely with the thickness of Co [21]. A Co thickness of $5 \mathrm{~nm}$ is chosen, thin enough to have a high exchange field and thick enough to promote (111) texture and square magnetization reversal [21]. In order to promote propagation of the acoustic waves into the $(\mathrm{Co} / \mathrm{IrMn})$ multilayer, 15 repetitions are stacked for a total thickness of $150 \mathrm{~nm}$. The multilayer stack is then annealed under in-plane magnetic field $(0.06 \mathrm{~T})$ for $1 \mathrm{~h}$ at $200{ }^{\circ} \mathrm{C}$ to set the exchange-bias direction (perpendicular to the $X$ direction of the LNO $41 Y$ cut).

The crystallographic quality of these sputtered samples is checked by conventional $(\vartheta-2 \vartheta) \mathrm{x}$-ray scattering experiments $(\mathrm{Cu} K \alpha$ wavelength) probing the crystallographic planes parallel to the surface.

In the Ni-based device, the Ni layer is (111) textured with a small contribution of (200), (220), and (311) grains (Fig. 1). The rocking curve across the (111) Bragg reflection exhibits a full width at half maximum of approximately $2.1^{\circ}$, which is a sign of relatively good crystallographic quality.

For the $(\mathrm{Co} / \mathrm{IrMn})$ multilayer, both $\mathrm{Co}$ and $\mathrm{IrMn}$ are (111) textured (Fig. 2). This texture is the one expected to

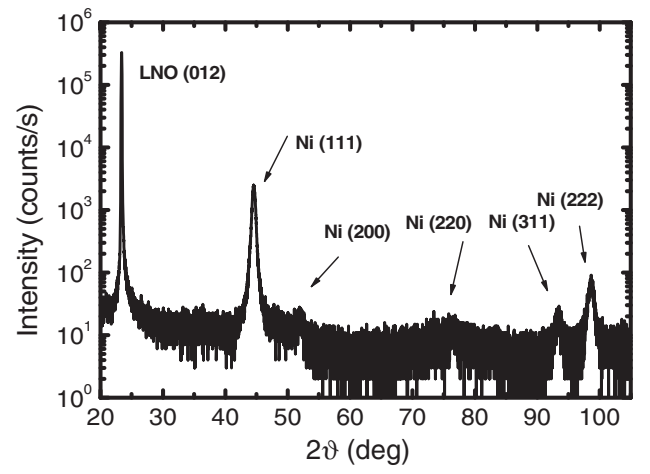

FIG. 1. Large-angle x-ray scattering specular scan for LNO/ $\mathrm{Ta}(5 \mathrm{~nm}) / \mathrm{Ni}(50 \mathrm{~nm}) / \mathrm{Ta}(5 \mathrm{~nm})$. 


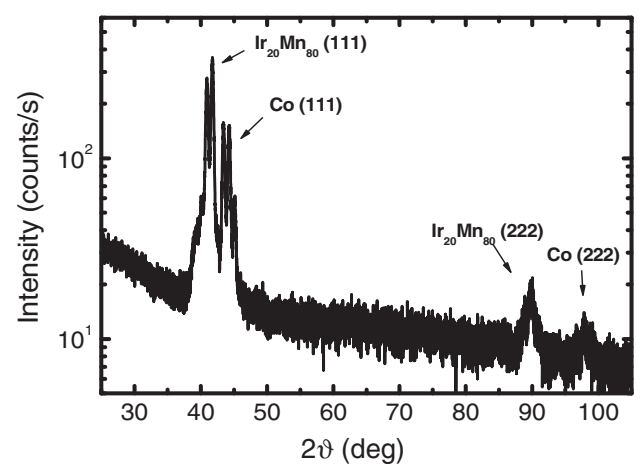

FIG. 2. Large-angle $\mathrm{x}$-ray scattering scan for $\mathrm{LNO} / \mathrm{Ta}(5 \mathrm{~nm}) /$ $\left[\mathrm{Co}(5 \mathrm{~nm}) / \mathrm{Ir}_{20} \mathrm{Mn}_{80}(5 \mathrm{~nm})\right]_{15} / \mathrm{Pt}(5 \mathrm{~nm})$.

permit us to get a high exchange field [21]. The multiple peaks around those attributed to the Co and IrMn layers are linked to the fact that the entire multilayer acts as a coherent entity, and their positions can be theoretically reproduced considering and computing the multiple reflections of the $\mathrm{x}$ rays at each atomic plane in a $(\mathrm{Co} / \mathrm{IrMn})$ multilayer [22].

The $\mathrm{TbFe}_{2}$-based heterostructures consist of 50-nm-thick $\mathrm{TbFe}_{2}$ films grown by molecular beam epitaxy (base pressure $5.10^{-11}$ mbar) on LNO 41Y-cut substrates. A 13-nm-thick Mo buffer is first deposited at $700^{\circ} \mathrm{C}$ both to isolate the $\mathrm{TbFe}_{2}$ compound from the oxide substrate and to limit possible $\mathrm{Li}$ diffusion $[20,23]$. $\mathrm{Tb}$ and $\mathrm{Fe}$ are then coevaporated from effusion cells in stoichiometric proportions, while keeping the substrate temperature around $700^{\circ} \mathrm{C}$. A 5-nm-thick Au layer is then deposited to protect the layer against oxidation and technological process. A full description of the sample growth and its structural characterization will be published elsewhere [20]. The LNO 41Y-cut substrate is a rather atypical template for crystalline growth since the physical surface does not correspond to any specific crystallographic planes. The (012) planes are tilted by approximately $-9^{\circ}$ with respect to the physical surface, the [0001] axis being tilted by $+50^{\circ}$. Extended reflection high-energy electron-diffraction (RHEED) and x-ray analysis interestingly reveal that the relative Mo and LNO lattice's orientation achieved on this specific cut is, nevertheless, similar to the one observed on the LNO Z cut: Mo (110) planes are parallel to LNO (0001) planes (i.e., thus, tilted by $50^{\circ}$ with respect to the surface), and two domains rotated by $60^{\circ}$ develop in this $M o(110)$ plane with the Mo [001] direction parallel to two equivalent LNO $[10-10]$ directions. The RHEED analysis performed during $\mathrm{TbFe}_{2}$ deposition attests to the crystalline growth, but up to now, $\mathrm{x}$-ray experiments could not permit us to unravel the exact orientation of the $\mathrm{TbFe}_{2}$ lattice and/or discuss the possible presence of different domains (see Fig. 3). Indeed, no peak can be identified on a specular x-ray scan which is directly linked to the tilted growth of the crystalline structure. While experiments and analysis are still in progress, we can infer a complex magnetic response of the $\mathrm{TbFe}_{2}$ layer and especially a three-dimensional distribution of magnetization during magnetization reversal.

\section{SENSOR STRUCTURE}

A resonator geometry is chosen to allow wireless measurements of the applied magnetic fields. The sensor structure is designed using a synchronous single-port SAW resonator, whose schematic is depicted in Fig. 4(a). The wavelength $(\lambda)$ is chosen equal to $6.5 \mu \mathrm{m}$, which is
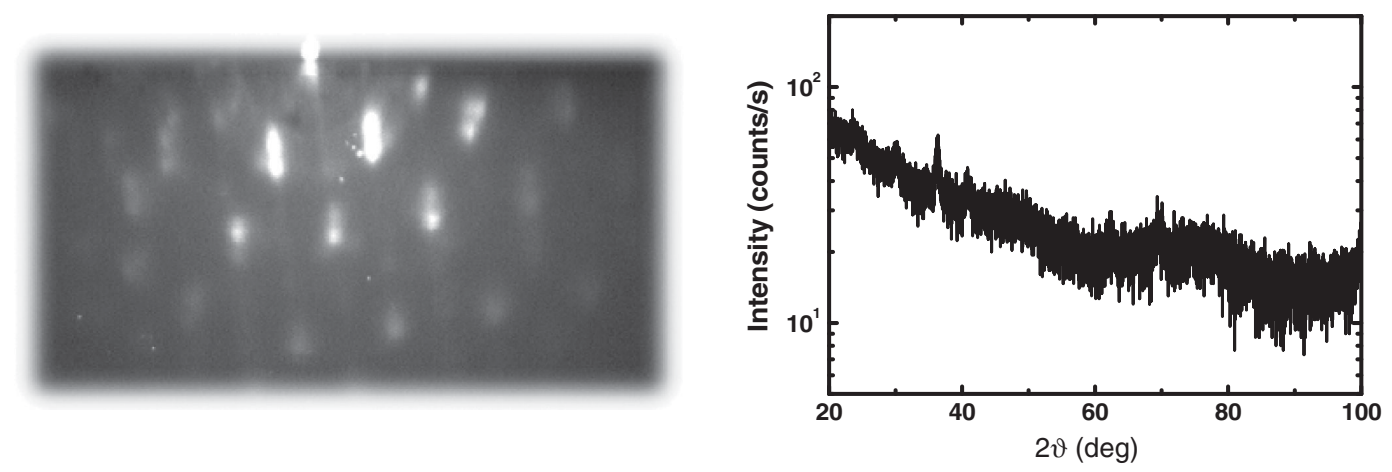

FIG. 3. Left: Reflection high-energy electron diffraction on the $\mathrm{TbFe}_{2}$ layer at $200^{\circ} \mathrm{C}$. Right: Large-angle $\mathrm{x}$ ray scattering on $\mathrm{TbFe}_{2}$ $50 \mathrm{~nm}$ on LNO $41 \mathrm{Y}$ cut.
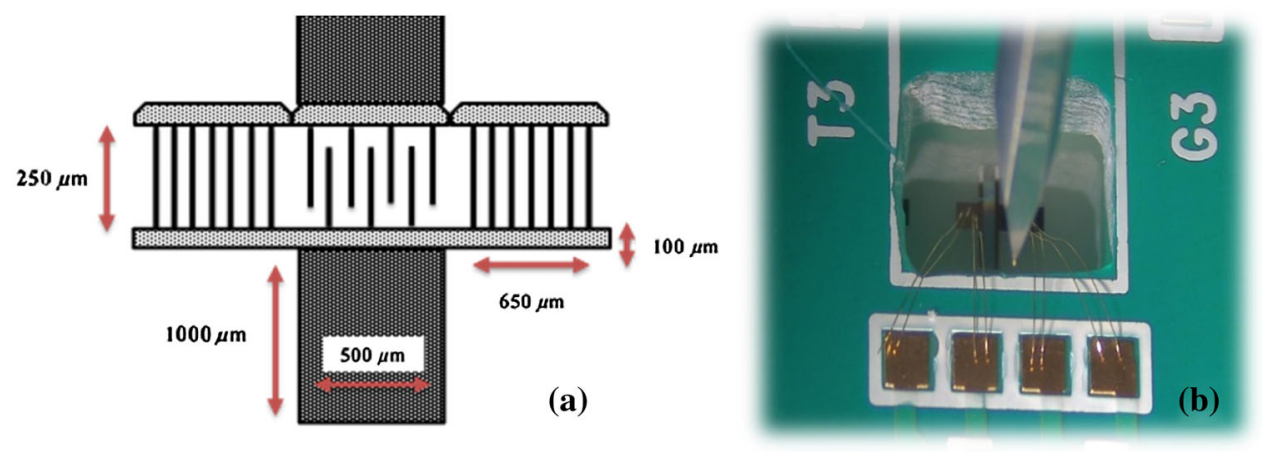

FIG. 4. (a) Schematic view of the magnetic sensor in a SAW resonator geometry. (b) SAW bonded on a chip for measurements under magnetic field. 
the minimum achievable with our photolithography equipment. The frequency resonances, thus, range between 400 and $800 \mathrm{MHz}$, depending on the substrate cut and/or acoustic wave mode considered. As we see in the following, the reduced width of the SAW fingers can have an impact on the magnetic properties of the magnetostrictive material and, thus, on the magnetic response of the SAW device. Depending on the magnetic material, the thickness of the IDTs varies between $0.007 \lambda$ and $0.025 \lambda$. The number of finger pairs is 100 , while the reflectors have 200 fingers.

The IDTs are fabricated from the continuous film using optical lithography and Ar ion etching. After film deposition, a positive resist is spin coated on the sample surface. After insolation through an optical mask, the SAW shape is transferred to the photoresist layer. This pattern is subsequently transferred to the magnetic layer using $\mathrm{Ar}$ ion etching. The etching process is followed using second ion mass spectrometry, and etching is stopped in the LNO substrate. The orientation of the IDTs is such that induced acoustic waves propagate along the $X$ crystallographic axis of the LNO $41 \mathrm{Y}$-cut substrate. It is important to underline that the $Y$ and $Z$ directions used in the following do not correspond to crystallographic axis. $Y$ corresponds to the in-plane direction perpendicular to the $X$ axis, and $Z$ corresponds to the direction perpendicular to the sample plane.

The frequency responses of the resonators are measured using a network analyzer (Agilent PNA 5230A, Santa Clara, CA) and a rf probe station (PM5, Suss Microtec). Table I summarizes the characteristics obtained for the resonators based on the different magnetic materials. For each device, a Rayleigh $(R)$ wave is identified at frequency around $550 \mathrm{MHz}$. The quality factor $(Q)$ and the electromechanical coupling coefficient $\left(K^{2}\right)$ values are also given. The product $Q K^{2}$ remains roughly constant and, in any case, sufficiently large to envision wireless interrogation. In order to prepare the test samples for magnetic characterization, the resonators are bonded to a copper-coated printing circuit board (PCB) suitable for microelectronics applications [Fig 4(b)]. The connections are established between the PCB and SAW device using a $20-\mu$ m-diameter gold wire. For all measurements presented in this paper, the intensity of the PNA transmitted signal is fixed to $0 \mathrm{~dB}$. It is checked that the shape of the response does not evolve as a function of intensity, i.e., that the device is working in its linear regime.

TABLE I. Characteristics of various SAW devices.

\begin{tabular}{lccc}
\hline \hline Magnetic material & $F(\mathrm{MHz})$ & $Q$ (arb. units) & $K^{2}(\%)$ \\
\hline $\mathrm{Ni}$ & 555 & 1100 & 0.23 \\
$\mathrm{TbFe}$ & 552 & 392 & 0.64 \\
$(\mathrm{Co} / \mathrm{IrMn})$ & 536.5 & 1101 & 0.2 \\
\hline \hline
\end{tabular}

\section{MAGNETIC CHARACTERISTICS AND ACOUSTIC WAVE RESPONSE}

Magnetic characterization of the magnetic layers is performed on full films before processing the resonator geometry using a vibrating superconducting quantuminterference-device magnetometer (VSM) and for magnetic fields applied along the in-plane $X$, in-plane $Y$, and perpendicular-to-the-plane $Z$ directions. Vectorial magnetometry is also undertaken to probe the field dependence of the in-plane magnetization components for fields applied perpendicular to the film plane.

The magnetoacoustic response of the processed SAW devices is characterized versus the magnetic field intensity and direction (in-plane $X$, in-plane $Y$, and perpendicular-tothe-plane $Z$ ) with IDTs made from the three types of sensitive magnetic layers $\left[\mathrm{Ni}, \mathrm{TbFe}_{2}\right.$, and $\left.(\mathrm{Co} / \mathrm{IrMn})\right]$. The IDTs are structured in order to make $X$ parallel to the SAW propagation direction (i.e., perpendicular to the IDTs). The resonance frequency $\left(f_{r}\right)$ determined from the $S_{11}$ response (Table I) is roughly equal to $550 \mathrm{MHz}$ and is in good agreement with the predictions considering the acoustic velocity of the Rayleigh wave $\left(V_{R}\right)$ in the $X$ direction of the LNO 41Y-cut substrate [19] and the acoustic wavelength $\left(f_{r}=V_{R} / \lambda\right)$.

\section{A. Ni-based SAW resonator}

As already mentioned, $\mathrm{Ni}$ is used as a starting point in this study as magnetostrictive material since various results using $\mathrm{Ni}$ as electrodes have been reported, including resonator geometries with a wireless response [9-11].

The magnetic response of the $\mathrm{Ni}$ thin film before processing is reported in Figs. 5(a) and 5(b). It appears that the easy magnetization axis of the Ni film lies along the inplane $X$ direction, with an anisotropy field of $9.4 \mathrm{mT}$. An easy axis of magnetization is often induced during sputtered film deposition either linked to the geometrical effects and/or stray field of the magnetron [24]. Along the perpendicular-tothe-plane $Z$ direction, a saturation field of $0.635 \mathrm{~T}$ can be measured. The extracted saturation magnetization $M_{S}=$ $505 \mathrm{kA} / \mathrm{m}$ is close to the expected value of $490 \mathrm{kA} / \mathrm{m}$ [25].

The device magnetoacoustic response for the Rayleigh wave is shown in Figs. 5(e) and 5(f) for the peak measured at $555 \mathrm{MHz}$ [Fig. 5(c)] and for different directions of the applied field [Fig. 5(d)]. The measured in-plane magnetoacoustic responses [Fig. 5(e)] are significantly different for the two directions, with a hysteretic behavior along the fingers of the IDTs and a reversible one along the SAW propagation direction. The shapes are rather similar to those previously reported on resonators $[9,10]$. Opposite responses can, however, be observed in delay lines where a full film with easy magnetization axis along $X$ is used [13]. While the hysteretic behavior can be problematic and, therefore, prohibit the development of magnetic field sensors, it has been hidden in most of the studies [9-11]. Indeed, only one 

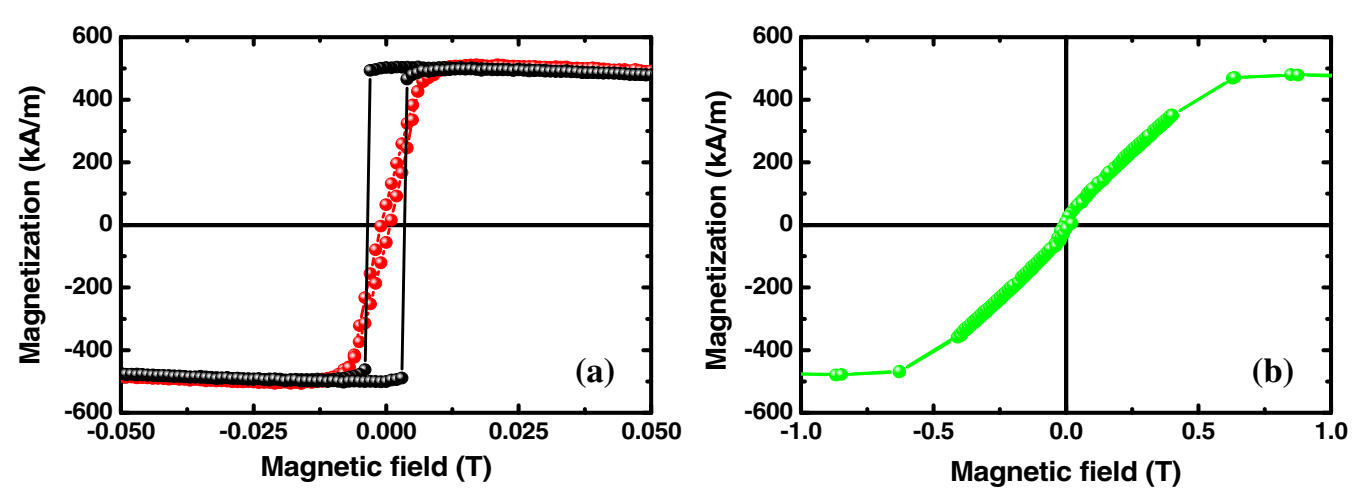

FIG. 5. Magnetic response of the Ni film deposited on LNO versus applied field along $X$ [(a) black], $Y$ [(a) red], and $Z$ [(b) green]. (c) $S_{11}$ versus frequency for the Rayleigh wave of the Ni-based resonator device. (d) Schematic of the resonator structure and definition of $X, Y$, and $Z$. Normalized SAW frequency variation of the
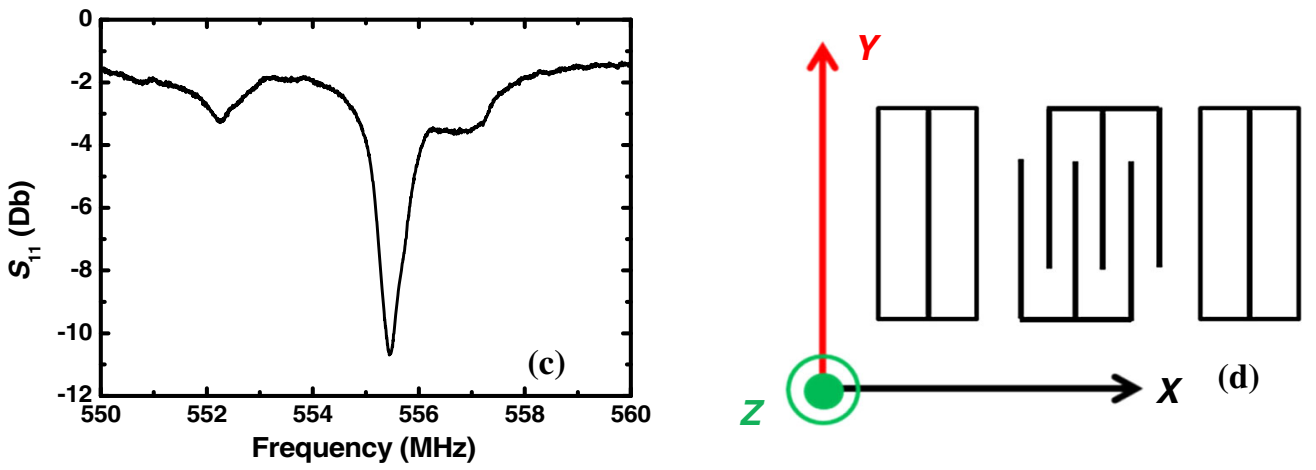

Ni-based resonator device versus applied field along $X$ [(e) black], $Y$ [(e) red], and $Z$ [(f) green]. (g) Normalized SAW frequency variation versus applied field along $Y$ (red) and successive minor loops for fields smaller than the coercive field.
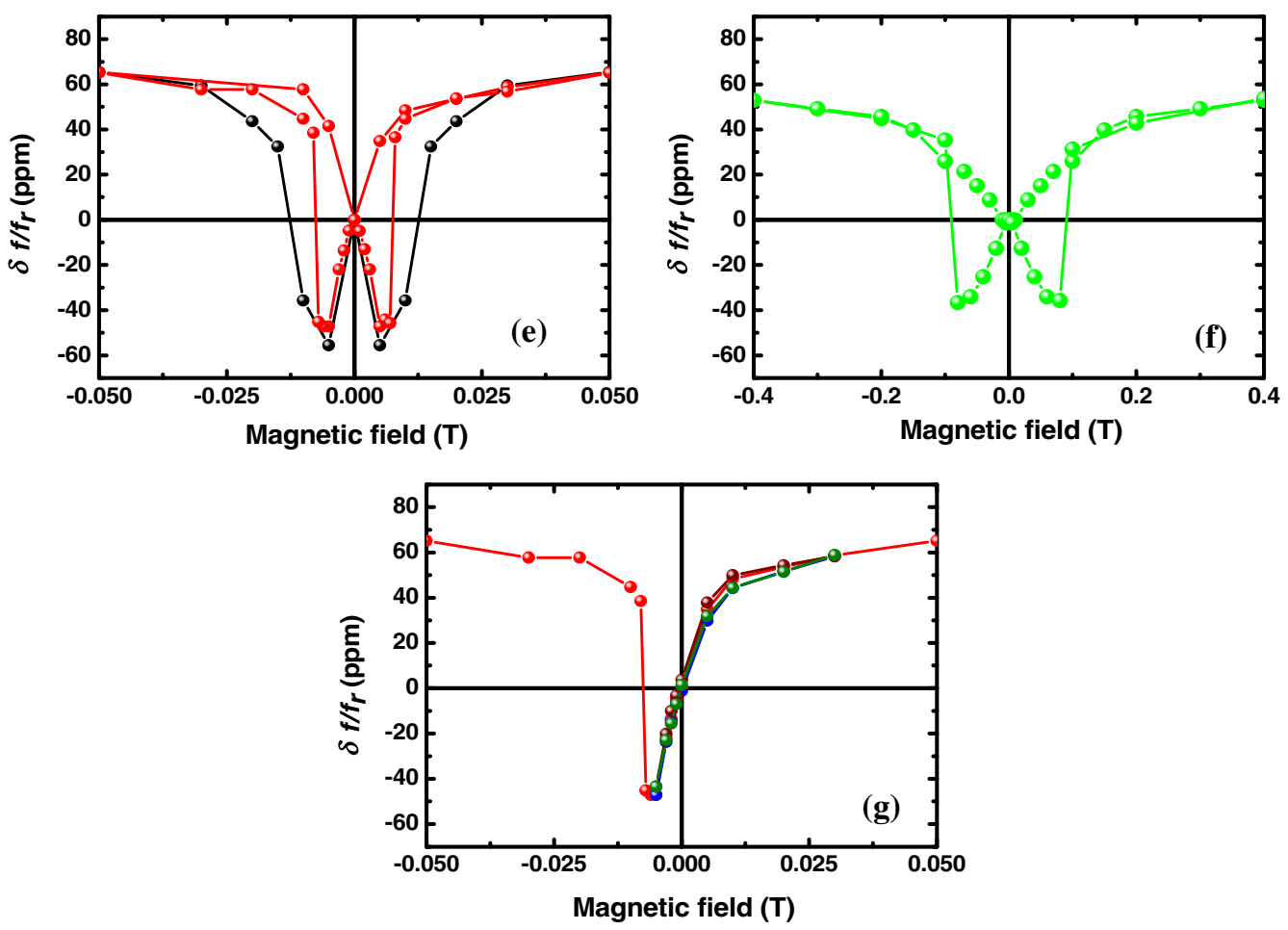

branch of the response is reported, or the field step is too large to observe the hysteresis, or the coercivity of the layer was too small to be observed.

Since the magnetic behavior of the SAW fingers is the basis to control the SAW magnetic response, anisotropic magnetoresistance (AMR) measurements are undertaken to directly explore the magnetic response of the wire [26].
The resistance of a magnetic material depends on the angle between the magnetization and the applied current with a squared cosine variation. Along the easy magnetization axis, no resistance variation is expected, while along the hard axis, the resistance should present a squared field variation. The AMR response measured on the SAW reflectors is reported in Fig. 6(a) when the field is applied 

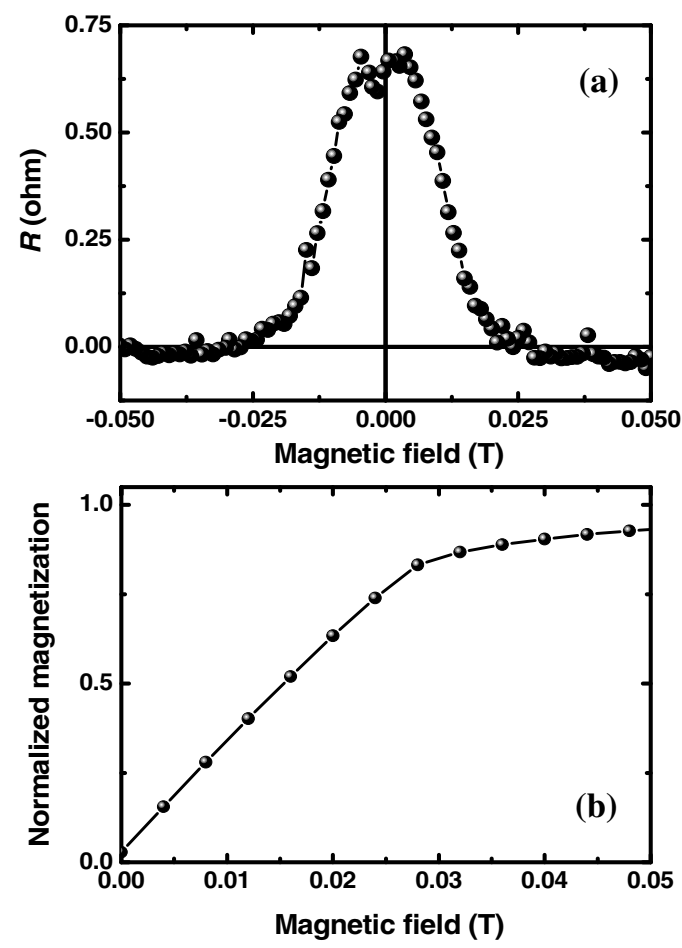

along $X$. In contrast to the results obtained for the full film before processing, $X$ appears to be the hard axis, and the easy axis of magnetization is, thus, parallel to the interdigitate fingers. As shown in Fig. 6(a), an anisotropy field of around $25 \mathrm{mT}$ is introduced by a shape effect and beats the natural anisotropy field of $9.4 \mathrm{mT}$ of the thin layer. Etching the film into wires, thus, induces the reorientation of the easy axis from $X$ towards $Y$.

In order to explain this effect, a micromagnetic calculation is performed for a $10-\mu \mathrm{m}-$ long, $1-\mu \mathrm{m}$-wide, and 50 -nm-thick Ni wire with cells size of $5 \mathrm{~nm}$. We use the magnetic parameters extracted from the VSM measurements and an exchange constant from the OOMMF simulator [27]. It appears clearly from the magnetic response with the field applied in plane and perpendicular to the wire length [Fig. 6(b)] that after saturation, the magnetization reorients towards the finger length at zero applied field. The saturation field calculated from the OOMMF simulation, $28 \mathrm{mT}$, is consistent with the value extracted from the AMR measurement. In our geometry, this shape anisotropy gives the opportunity to control the response when the field is applied in plane and perpendicular to the fingers. Changing the aspect ratio of the fingers leads to a change of this saturation field, and the SAW response can, thus, be interestingly tailored by the shape (aspect ratio) of the electrodes.

This first conclusion is of importance since, in the SAW resonator geometry, the response of the MSAW can be engineered by the IDT's shape. Indeed, up to now, the MSAW responses could not be engineered since their responses depend on the properties of the films that have been used, which are difficult to change or adjust. Here,
FIG. 6. (a) AMR measured on the Ni reflector wires when the field is applied in plane, perpendicular to the wire length. (b) Normalized magnetization versus applied field for a $\mathrm{Ni}$ wire calculated using OOMMF simulation. Field is applied in plane, perpendicular to the wire length, Micromagnetic configurations calculated using OOMMF for applied fields of $0.2 \mathrm{~T}$ (c), $0.04 \mathrm{~T}$ (d), $0.02 \mathrm{~T}$ (e), and $0 \mathrm{~T}$ (f).

we show that keeping the material constant, the MSAW response can be adjusted just by changing the aspect ratio of the IDTs. This feature is fundamental to adjust the MSAW characteristic for dedicated applications and opens the use of the MSAW in many applications for which the intensity of the field has to be measured, without hysteresis. From the technical point of view, the in-plane demagnetization factor perpendicular to the IDT's length $N_{x x}$ can be calculated using conventional magnetostatics or using micromagnetic codes. Then, the saturation field is simply computed using

$$
H_{D}=\mu_{0} N_{x x} M_{S}
$$

where $\mu_{0}$ is the permeability of free space, and $M_{S}$ is the saturation magnetization of the magnetic material.

As mentioned previously, when the field is applied along the easy axis of magnetization ( $Y$, i.e., along the IDTs), the presence of the hysteresis limits the use of the device as a field sensor, since for one applied field, different magnetic states can be stabilized and different SAW frequencies can be measured. However, for applied fields smaller than the coercive field, a bipolar response of the device can be interestingly obtained. Considering a square reversal of the magnetization, before reversal, the SAW response is reversible when measuring minor field loops [Fig. 5(g)]. As a consequence, increasing the coercivity of the materials should allow us to get a bipolar response for higher fields and over a wider magnetic field range.

The magnetoacoustic response is finally investigated for magnetic fields applied along the perpendicular-to-the-plane $Z$ direction [Fig. 5(f)]. This geometry is rarely reported in 


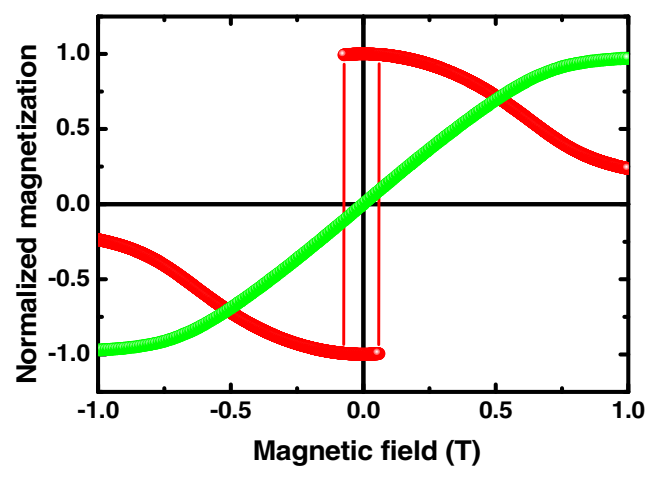

FIG. 7. Magnetic response of a Ni wire (along the field, green; perpendicular to the field, red) computed when the field is applied along $Z, 5^{\circ}$ tilted from $Z$ and $5^{\circ}$ tilted from $Y$. A macrospin model is used with the micromagnetic parameters extracted from magnetization measurements and shape anisotropy calculated using OOMMF.

previous works, and the response is not analyzed on the basis of the magnetic response of the magnetic wires. Surprisingly, while the magnetic response in this configuration is not hysteretic [Fig. 5(b)], the SAW response presents a pronounced hysteresis with a sharp reversal around $100 \mathrm{mT}$ [Fig. 5(f)]. We show that this hysteresis, in fact, relies on the behavior of the in-plane components of the magnetization. Indeed, the SAW response is sensitive to all components of magnetization [13], and the behavior of the in-plane components is, thus, required for a complete understanding of the magnetoacoustic response. Using a macrospin model and the magnetic parameters extracted from the magnetization curve (saturation magnetization and demagnetization field), the magnetization response is calculated when the field is tilted by $5^{\circ}$ from $Z$ and by $5^{\circ}$ from $Y$. While the calculated $Z$ component follows the experimental response measured with VSM [Fig. 7, green curve and Fig. 5(b)], the component along $Y$ clearly presents a steep reversal (Fig. 7, red curve) corresponding to the field for which a steep change of SAW frequency occurs [Fig. 5(f)]. This variation is of the same amplitude as the one observed during the steep magnetization reversal when the field is applied in plane, along $Y$ [Fig. 5(e), red curve]. Once again, this hysteresis limits the use of this geometry to build up a field sensor; a strategy has to be developed to control the in-plane magnetization component and suppress this sharp magnetization reversal.

In the following sections, we address the irreversibilities highlighted so far and try to find ways to remove or use them to build up unipolar or bipolar magnetic sensors. In a first step, we show that in the perpendicular geometry, the control of the in-plane component of magnetization is the key to remove hysteresis. In a second step, we show how increasing the coercive field of the thin layer allows us to get a bipolar response of the device for applied fields up to $0.5 \mathrm{~T}$.

\section{B. (Co/IrMn)-based system}

The association of ferromagnetic and antiferromagnetic materials, commonly encountered in spintronics devices [28], can provide interesting features to the development of MSAW devices. In our case, we use the first-order anisotropy induced by the exchange field to force the magnetization rotation in one sense. This rotation should suppress the jump observed in the in-plane magnetization component and, thus, in the perpendicular-to-the-plane response of the SAW, as observed in Ni-based devices.

The magnetic response of the $(\mathrm{Co} / \mathrm{IrMn})_{15}$ multilayer is presented in Fig. 8(a). The results show that the magnetization of the $(\mathrm{Co} / \mathrm{IrMn})_{15}$ multilayer lies in plane with an easy magnetization axis along $Y$ induced by the thermal annealing. The exchange field of $33.1 \mathrm{mT}$ is in good agreement with results reported in earlier work [21].

Along the perpendicular-to-the-plane $Z$ direction [Fig. 8(b)], a saturation field of $1.62 \mathrm{~T}$ can be measured leading to a saturation magnetization value of $M_{S}=1289 \mathrm{kA} / \mathrm{m}$, slightly lower than the expected value of $1420 \mathrm{kA} / \mathrm{m}$. The measurement of the in-plane magnetization components (red and black curves) confirms that the magnetization reversal does not exhibit any abrupt switch for magnetic fields applied along $Z$. Using a macrospin model and the magnetic parameters extracted from the magnetization curve (saturation magnetization, demagnetization field, and exchange field), we compute the magnetic response for the three magnetization components [continuous lines in Fig. 8(b)]. The main trends are well reproduced and confirm that no in-plane switch of magnetization is expected for perpendicular applied fields smaller than $2 \mathrm{~T}$ with a tilt of $\pm 5^{\circ}$.

The device magnetoacoustic response for the Rayleigh wave is shown in Figs. 8(e) and 8(f) for the peak measured at 536.5 MHz [Fig. 8(c)]. Two peaks are visible on that figure, likely due to parasitic interferences with the reflectors, but the field dependences are the same for the two waves. The measured variations of frequency versus inplane field are consistent with the results reported in the literature, considering an easy magnetization axis along $Y$. In this case, the shape anisotropy related to the resonator fingers adds to the anisotropy induced by the exchange bias. The presence of exchange bias just shifts the SAW response from the exchange-bias field, in agreement with the VSM response.

The SAW response measured in the $Z$ direction [Fig. 8(f)] confirms that controlling the in-plane rotation of magnetization suppresses the hysteretic behavior observed for the Ni-based device [Fig. 5(f)]. The SAW response is measured for tilt angles of the applied field up to $5^{\circ}$ from the perpendicular direction, and no hysteresis is detected in the response.

The goal is, thus, reached although the frequency variation under field remains relatively small. This can be linked to magnetostrictive coefficients in IrMn that are unknown 

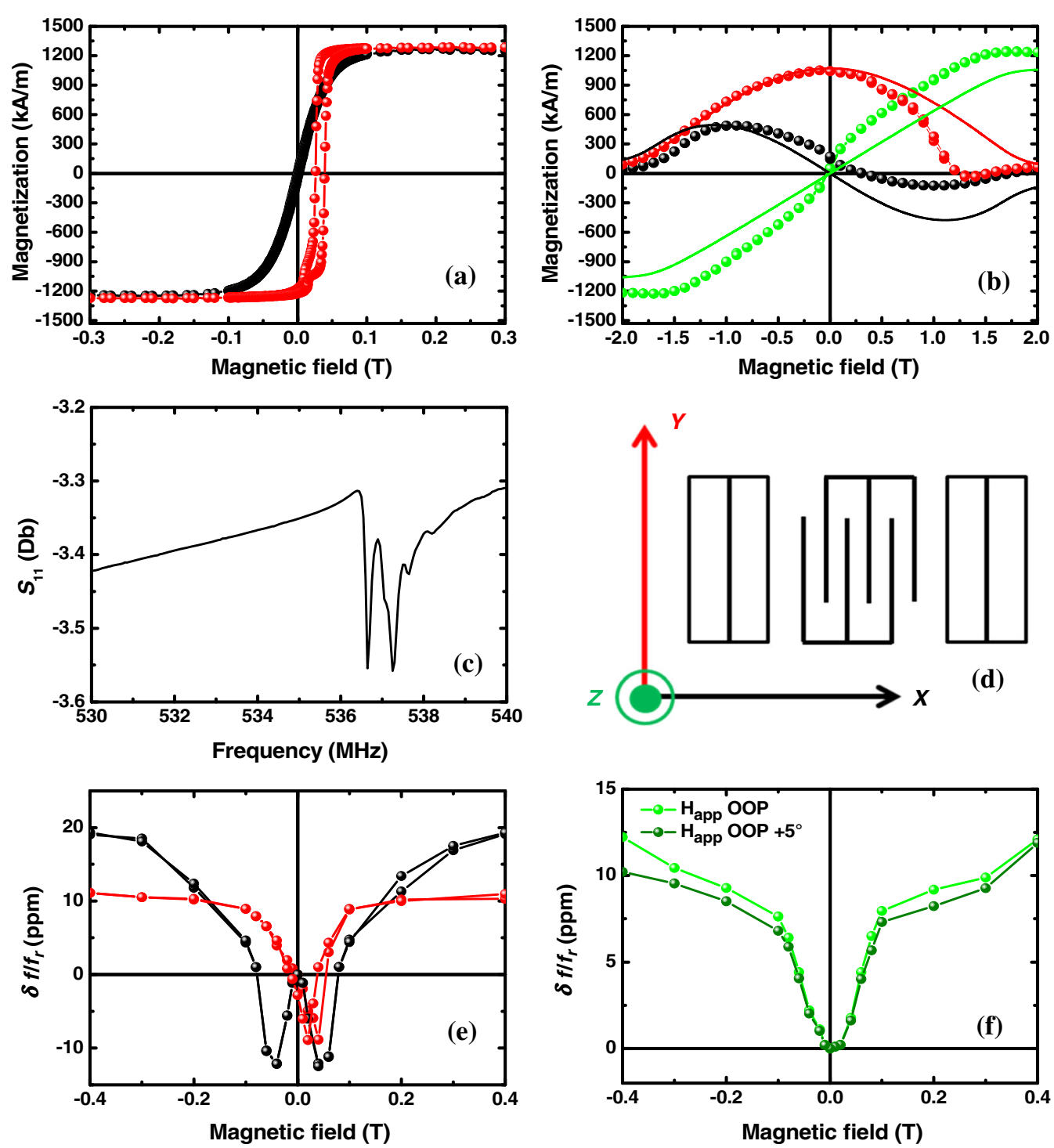

FIG. 8. Magnetic response of the $(\mathrm{Co} / \mathrm{IrMn})$ multilayer deposited on LNO versus applied field along $X$ [(a) black], $Y$ [(a) red], and $Z$ [(b) green]. In (b) are also plotted the magnetization components along $Y$ (red) and $X$ (black) for fields applied along $Z$. The continuous lines are calculated using a macrospin model. (c) $S_{11}$ versus frequency for the Rayleigh wave of the $(\mathrm{Co} / \mathrm{IrMn})$-based resonator device. (d) Schematic of the resonator structure and definition of $X, Y$, and $Z$. Normalized SAW frequency variation versus applied field along $X$ [(e) black], $Y$ [(e) red], and $Z$ [(f) green]. In (f), a measurement with the field applied $5^{\circ}$ off the perpendicular direction (dark green) is also presented. and cannot be found in the literature. In the future, other antiferromagnetic materials will be tested, especially those hosting frustration and exhibiting high magnetostrictive constants [29,30]. Engineering the multilayer stack can also improve the signal, as reported previously [13].

\section{C. $\mathrm{TbFe}_{2}$-based system}

$\mathrm{TbFe}_{2}$ thin films are also chosen as magnetostrictive material. $\mathrm{TbFe}_{2}$ has been used in previous studies [12] but always in its amorphous phase, which does not show the record saturation magnetostriction of the single-crystal compound. Even if the best crystalline quality is not achieved on the LNO 41Y cut (see Ref. [20] for other LNO orientations), the occurrence of a high magnetic coercivity is used for our demonstration.

The magnetic response of the $\mathrm{TbFe}_{2}$ thin film [Figs. 9(a) and 9(b)] shows that the magnetization reversal process in $\mathrm{TbFe}_{2}$ is much more complex than the one in $\mathrm{Ni}$ and (Co/IrMn) films. Despite a square response measured along $Y$ showing that the easy magnetization axis is mainly along this direction, a hysteresis opening in the response along $X$ and $Z$ [Fig. 9(b)] is the sign of a complex 3D distribution. Along $Y$, a coercivity of $0.56 \mathrm{~T}$ is measured, 60 times larger than in Ni samples. The saturation magnetization measured by VSM has a value close to the theoretical value of $M_{S}=800 \mathrm{kA} / \mathrm{m} \mathrm{[20]}$.

The device magnetoacoustic response for the Rayleigh wave is presented in Figs. 9(e) and 9(f) for the peak measured at $552 \mathrm{MHz}$ [Fig. 9(c)]. As for the (Co/IrMn)based device, the shape anisotropy of the resonator fingers adds to the strong anisotropy of the full film, and the easy magnetization axis is the same in the film and in the device. The response along this easy axis is consistent with the results obtained for Ni-based and (Co/IrMn)-based devices. Note, however, that a hysteretic response is also observed along $X$, consistent with the hysteretic magnetization reversal along this direction [Fig. 9(a), black curve]. Despite those irreversible behaviors, the increased coercive 

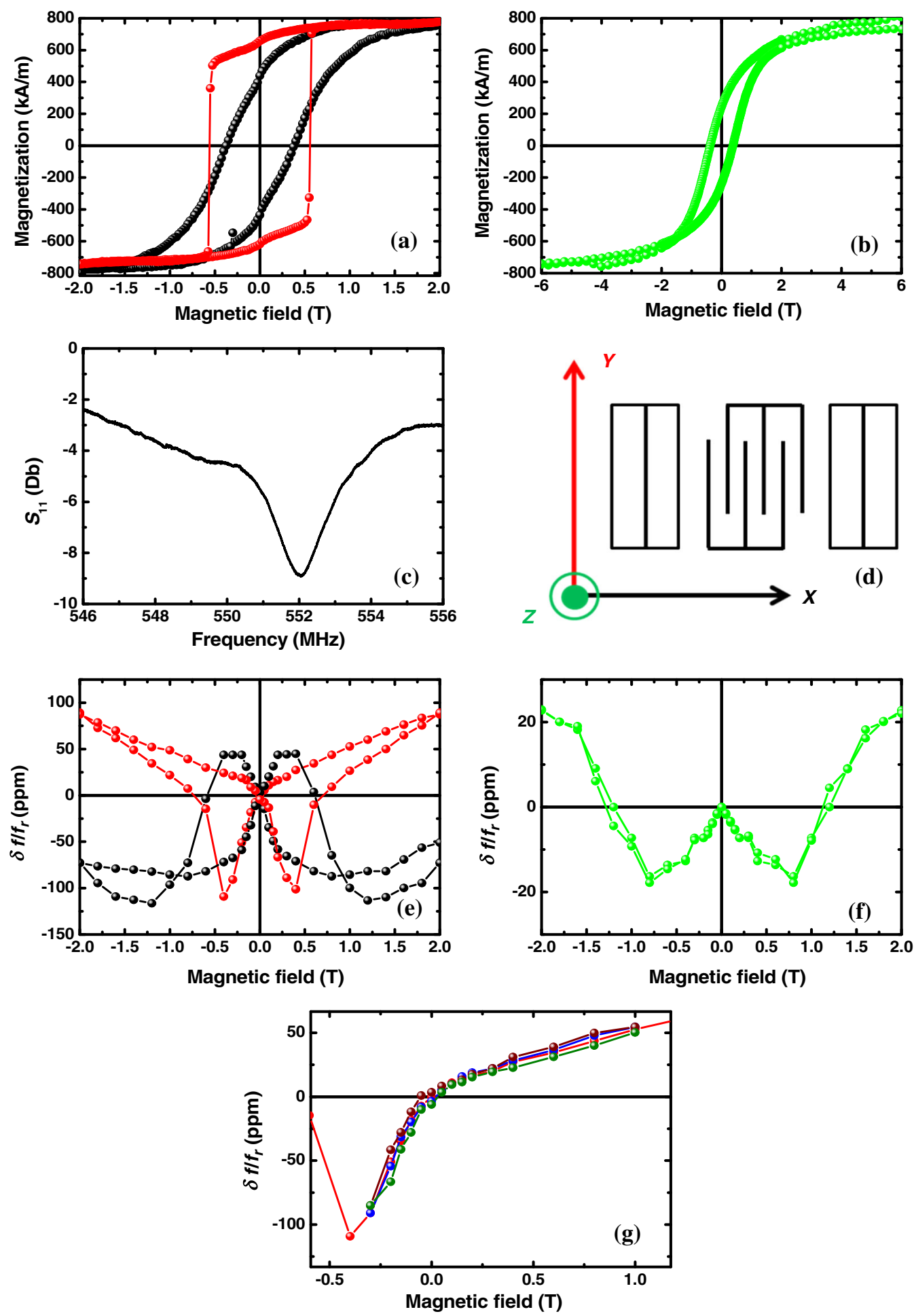

field compared with Ni-based devices leads to a significant extension of the magnetic field range where a bipolar response can be obtained. The SAW response remains reversible during the measurements of successive minor field loops, as long as the magnetic field does not reach coercivity [Fig. 9(g)], and the large coercivity, thus, allows us to get a bipolar response for high applied fields. One can finally underline the surprising reversible SAW response (d)
FIG. 9. Magnetic response of the $\mathrm{TbFe}_{2}$ film deposited on LNO versus applied field along $X$ [(a) black], $Y$ [(a) red], and $Z$ [(b) green]. (c) $S_{11}$ versus frequency for the Rayleigh wave of the $\mathrm{TbFe}_{2}$-based resonator device. (d) Schematic of the resonator structure and definition of $X, Y$, and $Z$. Normalized SAW frequency variation versus applied field along $X[(\mathrm{e})$ black], $Y$ [(e) red], and $Z$ [(f) green]. (g) Normalized SAW frequency variation versus applied field along $Y$ (red) and successive minor loops for fields smaller than the coercive field. 
measured for Ni-based devices. We believe that the main reason for this is the deterioration of the LNO surface when heated to $700{ }^{\circ} \mathrm{C}$ for the $\mathrm{TbFe}_{2}$-layer growth. A transmissionelectronic-microscopy study with chemical analysis shows that an approximately 60 -nm-thick $\mathrm{Li}_{2} \mathrm{O}$ layer forms at the surface [20]. From Comsol simulations, such a $\mathrm{Li}_{2} \mathrm{O}$ layer inserted between the substrate and the magnetic material induces a significant decrease of the electromechanical coupling and, thus, of the frequency variation [20].

\section{CONCLUSION}

We show in this study that the magnetic response of a magnetic SAW device is much more complex than often reported up to now and that a detailed understanding of the magnetic properties is essential to unravel and control the magnetoacoustic response.

The hysteretic behavior, a key issue for the development of magnetic field sensors, is especially addressed. The presence of hysteresis in the in-plane magnetoacoustic response appears to be directly linked to magnetic irreversibilities in the magnetic part of the device, i.e., to the in-plane anisotropy properties. In the SAW resonator geometry, the shape of the SAW fingers might play a significant role in the magnetic anisotropy, especially for soft magnetic materials. Engineering the IDT's shape can, thus, permit us to tailor and control the SAW field response. We show more generally that the proper choice of magnetic material and the control of the magnetic properties helps to build up high-field unipolar or bipolar field sensors. A bipolar magnetic field sensor response can be achieved for fields up to $0.5 \mathrm{~T}$.

Our investigation for magnetic fields perpendicular to the plane proves that the magnetoacoustic response is sensitive to the hysteretic behavior of in-plane magnetization components. Introducing ferromagnetic and antiferromagnetic bilayers, the elements commonly used in spintronic devices, hysteretic reversals can be suppressed in the perpendicular-to-film plane geometry. Additional work using high magnetostrictive antiferromagnets has to be undertaken.

\section{ACKNOWLEDGMENTS}

The authors thank Sylvie Robert for help with the $\mathrm{x}$-ray diffraction experiments. Experiments are carried out on IJL Project TUBE-Davm equipment funded by the FEDER (EU), French PIA project "Lorraine Université d'Excellence (Grant No. ANR-15-IDEX-04-LUE), Region Grand Est, Metropole Grand Nancy, and ICEEL.

[1] W. Buff, F. Plath, O. Schmeckebier, M. Rusko, T. Vandahl, H. Luck, F. Moller, and D. C. Malocha, Remote sensor system using passive SAW sensors, in Proceedings of IEEE Ultrasonics Symposium (Cannes, France, 1994), pp. 585-588.
[2] W. Buff, M. Rusko, T. Vandahl, M. Goroll, and F. Moller, A differential measurement SAW device for passive remote sensoring, in Proceedings of the Ultrasonics Symposium (San Antonio, TX, 1996), pp. 343-346.

[3] W. Buff, S. Klett, M. Rusko, J. Ehrenpfordt, and M. Goroll, Passive remote sensing for temperature and pressure using SAW resonator devices, IEEE Trans. Ultrason. Ferroelectr. Freq. Control 45, 1388 (1998).

[4] V. Kalinin, G. Brown, and A. Leigh, Contactless torque and temperature sensor based on SAW resonators, in Proceedings of IEEE International Ultrasonics Symposium (Vancouver, Canada, 2006) pp. 1490-1493.

[5] T. Aubert, O. Elmazria, B. Assouar, L. Bouvot, and M. Oudich, Surface acoustic wave devices based on AlN/sapphire structure for high temperature applications, Appl. Phys. Lett. 96, 203503 (2010).

[6] A. Talbi, F. Sarry, M. Elhakiki, L. Le Brizoual, O. Elmazria, P. Nicolay, and P. Alnot, ZnO/quartz structure potentiality for surface acoustic wave pressure sensor, Sens. Actuators 128, 78 (2006).

[7] N. Tiercelin, V. Preobrazhensky, P. Pernod, and A. Ostaschenko, Enhanced magnetoelectric effect in nanostructured magnetostrictive thin film resonant actuator with field induced spin reorientation transition, Appl. Phys. Lett. 92, 062904 (2008).

[8] N. Tiercelin, A. Talbi, V. Preobrazhensky, P. Pernod, V. Mortet, K. Haenen, and A. Soltani, Magnetoelectric effect near spin reorientation transition in giant magnetostrictivealuminum nitride thin film structure, Appl. Phys. Lett. 93, 162902 (2008).

[9] M. Kadota, S. Ito, Y. Ito, T. Hada, and K. Okaguchi, SAW magnetic sensors composed of various Ni electrode structures on quartz, in Proceedings of IEEE International Ultrasonics Symposium, 2011.

[10] M. Kadota, S. Ito, Y. Ito, T. Hada, and K. Okaguchi, Magnetic sensor based on surface acoustic wave resonators, Jpn. J. Appl. Phys. 50, 07HD07 (2011).

[11] D. C. Webb, D. W. Forester, A. K. Gangu1y, and C. Vittoria, Application of amorphous magnetic-layers in surfaceacoustic-wave devices, IEEE Trans. Magn. 15, 1410 (1979).

[12] M. Yamaguchi, K. Y. Hashimoto, H. Kogo, and M. Naoe, Variable saw delay line using amorphous $\mathrm{TbFe}_{2}$ film, IEEE Trans. Magn. 16, 916 (1980).

[13] H. Zhou, A. Talbi, N. Tiercelin, and O. Bou Matar, Multilayer magnetostrictive structure based surface acoustic wave devices, Appl. Phys. Lett. 104, 114101 (2014).

[14] A. K. Ghosh, Introduction to Measurements and Instrumentation (PHI Learning Pvt. Ltd. 2012), https://play.google.com/ store/books/details/ARUN_K_GHOSH_INTRODUCTION_ TO_MEASUREMENTS_AND_INST?id=k58cIAoI3YIC $\& \mathrm{hl}=\mathrm{en}$.

[15] A. E. Clark, Magnetostrictive rare earth $-\mathrm{Fe}_{2}$ compounds, Naval Surface Weapons Center, White Oak Laboratory, 1977.

[16] E. du Trémolet de Lacheisserie, Magnétisme: II-Matériaux et applications (Presses Universitaires de Grenoble, Grenoble, France, 1999).

[17] J. P. Nozières, S. Jaren, Y. B. Zhang, K. Pentek, A. Zeltser, P. Wills, and V.S. Speriosu, Correlation between lifetime 
and blocking temperature distribution in spin-valve structures, Am. Inst. Phys. 87, 6609 (2000).

[18] S. U. Jen, Y. D. Yao, Y. T. Chen, J. M. Wu, C. C. Lee, T. L. Tsai, and Y. C. Chang, Magnetic and electrical properties of amorphous CoFeB films, J. Appl. Phys. 99, 053701 (2006).

[19] C. K. Campbell, Surface Acoustic Wave Devices for Mobile and Wireless Communications (Academic Press, San Diego, 1998).

[20] V. Polewczyk, Ph.D. thesis, Lorraine University, 2017.

[21] G. Malinowski, Ph.D. thesis, Université H. Poincaré, 2004.

[22] G. Malinowski, M. Hehn, O. Lenoble, and A. Schuhl, Magnetic origin of enhanced top exchange biasing in Py/IrMn/Py multilayers, Phys. Rev. B 68, 184404 (2003).

[23] M. Huth and C. P. Flynn, Strain-induced perpendicular magnetization in $\mathrm{TbFe}_{2}(111)$ thin films on $\mathrm{LiNbO}_{3}$, J. Magn. Magn. Mater. 204, 204 (1999).

[24] E. Teng and N. Ballard, Anisotropy induced signal waveform modulation of DC magnetron sputtered thin film disks, IEEE Trans. Magn. 22, 579 (1986).
[25] H. Danan, A. Herr, and A. J. P. Meyer, New determinations of the saturation magnetization of nickel and iron, J. Appl. Phys. 39, 669 (1968).

[26] I. A. Campbell, A. Fert, and O. Jaoul, The spontaneous resistivity anisotropy in Ni-based alloys, J. Phys. C 3, S95 (1970).

[27] M. J. Donahue and D. G. Porter, National Institute of Standards and Technology Report No. NISTIR 6376, 1999.

[28] Y. F. Liu, J. W. Cai, W. Y. Lai, and G. H. Yu, Effect of Ir-Mn composition on exchange bias and thermal stability of spin valves with nano-oxide layers, J. Appl. Phys. 103, 093908 (2008).

[29] H. Gomonay and V. M. Loktev, Magnetostriction and magnetoelastic domains in antiferromagnets, J. Phys. Condens. Matter 14, 3959 (2002).

[30] W. Y. Peng and H. Zhang, Magnetostriction studies in an antiferromagnetic polycrystalline $\mathrm{Mn}_{42} \mathrm{Fe}_{58}$ alloy, Appl. Phys. Lett. 89, 262501 (2006). 